\title{
INTOXICAÇÃO EXPERIMENTAL POR NARASINA EM BOVINOS ${ }^{1}$
}

\author{
Angelica T. B. Wouters ${ }^{2}$, Flademir Wouters ${ }^{2}$ e Claudio S. L. Barros ${ }^{3}$
}

\begin{abstract}
Wouters A.T.B., Wouters F. \& Barros C.S.L. 1997. [Experimental narasin poisoning in cattle.] Intoxicação experimental por narasina em bovinos. Pesquisa Veterinária Brasileira 17(2):82-88. Depto Patologia, Universidade Federal de Santa Maria, RS 97119-900, Brazil.

Experimental poisoning by narasin was induced in 11 calves through oral administration of the drug. Five calves died, one was killed in extremis and 5 recovered after presenting mild clinical signs of the toxicosis. The onset of clinical signs was 12 hours to 4 days after the administration of the drug and clinical courses lasted from 24 hours to 9 days. Clinical signs were anorexia, decreased intensity and frequency of ruminal movements. Consistency of the feces varied from dry to semiliquid or liquid. Affected calves were reluctant to move, had incoordinated and stiff gait, and dragged the point of the hooves while walking. There was sluggish masticatory movements with drooling of saliva and dysphagia. The intensity of cardiac sounds were increased and, in one case, the jugular was distended and pulsating. Some animals were depressed, presented dyspnea, polydipsia and groanings. One calf presented myoglobinuria. Main necropsy findings were in the myocardium and consisted of focal or diffuse pale irregular areas. Signs of heart failure such as pulmonary edema, hydrotorax and clotted blood in the left ventricle of the heart were also observed. Degenerative lesions characterized by pale areas were seen in the skeletal muscles of three calves, but were marked in only one. Most affected muscles were masseter, extrinsec muscles of the tongue, tensor fasciae latae, semitendinosus, semimembranosus, psoas, quadriceps femoris, infraspinatus and gastrocnemius. Main histopathological changes consisted of degenerative myopathy and cardiomyopathy characterized by vacuolization of myofibers, hyalin and flocular necrosis either associated or not with reparative events. Myofiber regeneration and fibrosis were observed respectively in the skeletal muscles and in the myocardium.
\end{abstract}

INDEX TERMS: Narasin, ionophore antibiotic, cattle, pathology, toxicosis.

SINOPSE.- Intoxicação por narasina foi induzida pela administração oral da droga a 11 bovinos. Desses, cinco tiveram morte espontânea, um foi sacrificado in extremis e outros cinco apresentaram sinais clínicos da toxicose, mas se recuperaram. Os sinais clínicos iniciaram entre 12 horas e 4 dias após ingestão da narasina e a duração do curso clínico variou de 24 horas até 9 dias. Houve anorexia, redução na intensidade e número de movimentos ruminais, fezes pastosas, ressequi-

\footnotetext{
${ }^{1}$ Aceito para publicação em 2 de abril de 1997.
}

Parte da tese de mestrado do primeiro autor. Defendida no Curso de PósGraduação (CPG) em Medicina Veterinária, área de concentração em patologia, Universidade Federal de Santa Maria (UFSM), em 29 de janeiro de 1997. Trabalho financiado pelo CNPq, projeto 530198/93-2.

${ }^{2}$ CPG em Medicina Veterinária, área de concentração em patologia, Centro de Ciências Rurais, UFSM, 97119-900 Santa Maria, Rio Grande do Sul.

${ }^{3}$ Departamento de Patologia, UFSM. Bolsista do CNPq ( 350938/91-1). das ou diarréicas. Os animais apresentavam relutância em movimentar-se, tinham andar incoordenado e rígido, e caminhavam arrastando as pinças dos membros posteriores. Em alguns a mastigação era lenta, com salivação abundante e havia disfagia. A intensidade dos sons cardíacos estava aumentada e, em um caso, ocorreu discreto pulso positivo da jugular. Alguns animais apresentaram depressão, gemidos, dispnéia, respiração abdominal e polidipsia. Em um caso houve mioglobinúria. As principais alterações observadas à necropsia localizavam-se no miocárdio e consistiam de áreas pálidas irregulares focais ou palidez difusa. Sinais de insuficiência cardíaca como edema pulmonar, hidrotórax, edema subcutâneo de declive e sangue coagulado no ventrículo esquerdo foram também observados. Alterações degenerativas representadas por áreas pálidas foram vistas nos músculos esqueléticos de 3 bovinos, mas de forma marcada somente em um. Os músculos mais envolvidos foram masseter, extrínsecos da língua, tensor da fáscia lata, semitendíneo, 
semimembranáceo, psoas, quadriceps femoris, músculos da face medial da escápula, grande dorsal e gastrocnêmio. A histopatologia consistiu de miopatia e cardiomiopatia degenerativas. Regeneração de miofibras e fibrose foram observadas, respectivamente, nos músculos esqueléticos e no miocárdio.

TERMOS DE INDEXAÇÃO: Narasina, antibióticos ionóforos, bovinos, patologia, intoxicações.

\section{INTRODUÇÃO}

Antibióticos ionóforos formam complexos químicos bipolares com cátions facilitando o transporte iônico através de membranas biológicas, alterando o equilíbrio iônico intracelular. São usados principalmente como coccidiostáticos para aves e bovinos e como promotores de crescimento para bovinos (Novilla 1992). São considerados seguros quando usados nas espécies-alvo, dentro das dosagens recomendadas pelo fabricante. Uso inadequado de antibióticos ionóforos, tem, no entanto, causado intoxicações em várias espécies animais, como bovinos (Schweitzer et al. 1984, Geor \& Robinson 1985), equinos (Rollinson et al. 1987), ovinos (Bourque et al. 1986), suínos (Miskimins \& Neiger 1996), cães (Wilson 1980, Karsai et al. 1990), coelhos (Salles et al. 1994) e aves (Beck \& Harries 1979). As lesões associadas à intoxicação em todas essas espécies são caracterizadas por lesões degenerativas nos músculos esqueléticos e no miocárdio.

O uso terapêutico de antibióticos ionóforos em medicina veterinária difundiu-se muito nos últimos anos, com um consequente aumento no risco de intoxicação em animais. $O$ perigo potencial que representa a ocorrência de surtos adicionais pelo desconhecimento da intoxicação, motivou a realização deste estudo.

Este trabalho tem como objetivos determinar o quadro clínico, as lesões de necropsia e a histopatologia da intoxicação por narasina em bovinos.

\section{MATERIAL E MÉTODOS}

Doze bovinos, machos castrados, sem raça definida, com idades entre 6 e 10 meses foram divididos em seis lotes de dois animais e mantidos em baias de alvenaria. Durante todo o experimento receberam água fresca e feno de alfafa à vontade. Um composto contendo $10 \%$ de narasina ${ }^{4}$ diluído em água foi administrado a 11 desses bovinos por via oral (Quadro 1). Um bovino controle foi mantido durante todo o experimento sob as mesmas condições dos outros, não tendo recebido a droga.

Os bovinos eram submetidos a exame clínico pela manhã e no final da tarde. Diariamente eram retirados das baias e movimentados. Os animais que morreram espontaneamente ou foram sacrificados, incluindo o controle, foram submetidos a necropsia. Fragmentos dos músculos esqueléticos quadríceps femoral, semitendíneo, longissimus dorsi, diafragma, tríceps, supra-espinhal, ocular e abdominal, miocárdio, língua, encéfalo, pulmão, fígado,

${ }^{4}$ Monteban 100. Nutris Tecnologia e Sistema de Nutrição Ltda., BR 116, Km 73,5, Trevo Distrito Industrial, Quatro Barras, Paraná 83420-000.
Quadro 1. Delineamento experimental.da intoxicação experimental por narasina em bovinos

\begin{tabular}{|c|c|c|c|c|}
\hline Bovino & $\begin{array}{l}\text { Peso } \\
(\mathrm{kg})\end{array}$ & $\begin{array}{l}\text { Administração } \\
\text { diária (mg/kg) }\end{array}$ & $\begin{array}{c}\text { Número de } \\
\text { administrações }\end{array}$ & $\begin{array}{c}\text { Total administrado } \\
(\mathrm{mg} / \mathrm{kg})\end{array}$ \\
\hline 01 & 56 & 30 & 1 & 30 \\
\hline 02 & 110 & 15 & 2 & 30 \\
\hline 03 & 128 & 15 & 1 & 15 \\
\hline 04 & 101 & 7 & 3 & 21 \\
\hline 05 & 146 & 4 & 1 & 4 \\
\hline 06 & 109 & 7 & 1 & 7 \\
\hline 07 & 120 & 4 & 4 & 16 \\
\hline 08 & 130 & 2 & 7 & 14 \\
\hline 09 & 125 & 1 & 14 & 14 \\
\hline $10^{\mathrm{a}}$ & 140 & - & - & - \\
\hline 11 & 178 & 15 & 1 & 15 \\
\hline 12 & 90 & 7 & 4 & 28 \\
\hline
\end{tabular}

${ }^{\text {a }}$ Controle.

esôfago, rúmen, abomaso, intestino delgado, intestino grosso e linfonodos mesentérico e pré-escapular foram colhidos e processados rotineiramente para histopatologia. Cortes selecionados de músculo esquelético e de miocárdio foram corados pelo tricrômico de Masson.

As alterações histológicas dos músculos esqueléticos e do miocárdio foram classificadas em degenerativo-necróticas e reparativas; nas primeiras, foram incluídas vacuolização do sarcoplasma, necrose segmentar hialina e flocular da miofibra; nas alterações reparativas foram incluídas reações inflamatórias (incluindo fibrose) e regenerativas das miofibras.

\section{Sinais clínicos}

\section{RESULTADOS}

Dos 11 bovinos que receberam a droga, cinco morreram espontaneamente (bovinos 1, 2, 4, 11, 12), um (bovino 3) foi sacrificado in extremis e cinco (bovinos 5 a 9) recuperaram-se após apresentarem sinais clínicos leves e curso com

Quadro 2. Início e duração dos sinais clínicos após a administração experimental de narasina aos bovinos

\begin{tabular}{|c|c|c|c|}
\hline Bovino & $\begin{array}{l}\text { Início dos sinais clínico } \\
\text { após a primeira } \\
\text { administração }\end{array}$ & $\begin{array}{l}\text { Duração do curso } \\
\text { clínico }\end{array}$ & Desfecho \\
\hline 01 & $18 \mathrm{~h}$ & $6 d$ & $\mathrm{E}^{\mathrm{a}}$ \\
\hline 02 & $18 \mathrm{~h}$ & $42 \mathrm{~h}$ & $\mathrm{E}$ \\
\hline 03 & $60 \mathrm{~h}$ & $4 \mathrm{~d}$ e $12 \mathrm{~h}$ & $S^{b}$ \\
\hline 04 & $24 \mathrm{~h}$ & $6 \mathrm{~d}$ e $2 \mathrm{~h}$ & $\mathrm{E}$ \\
\hline 05 & $40 \mathrm{~h}$ & $24 \mathrm{~h}$ & $\mathrm{R}^{\mathrm{c}}$ \\
\hline 06 & $12 \mathrm{~h}$ & $48 \mathrm{~h}$ & $\mathrm{R}$ \\
\hline 07 & $36 \mathrm{~h}$ & $9 d$ & $\mathrm{R}$ \\
\hline 08 & $12 \mathrm{~h}$ & $2 d$ & $\mathrm{R}$ \\
\hline 09 & $4 d$ & $6 d$ & $\mathbf{R}$ \\
\hline $10^{d}$ & - & - & $\mathrm{S}$ \\
\hline 11 & $16 h$ & $9 d$ & $\mathrm{E}$ \\
\hline 12 & $3 d$ & $60 \mathrm{~h}$ & $\mathrm{E}$ \\
\hline
\end{tabular}


duração variando entre 1 a 9 dias (Quadro 2). O bovino controle não apresentou sinais clínicos, alterações de necropsia ou histopatológicas que pudessem ser relacionadas à toxicose.

Os sinais clínicos foram mais evidentes nos seis animais que morreram e serão descritos primeiro. Eram principalmente relacionados a disfunção muscular e a distúrbios digestivos. A maioria dos animais afetados relutava em andar (bovinos $3,4,11$ e 12), levantava somente quando forçada, (bovinos 1-4 e 12), deitando logo após levantar (bovinos 1, 4 e 12). $\mathrm{O}$ andar era incoordenado nos bovinos 2, 3, 11 e 12 (Fig. 1) e rígido (bovinos 3, 11 e 12). Os animais arrastavam as pinças dos cascos dos membros posteriores ao caminhar (bovinos 1-4, 11 e 12). Esse sinal clínico era particularmente pronunciado em três animais (bovinos 3,11 e 12). Ao se alimentarem, os bovinos mastigavam lentamente, por longo tempo, a mesma porção de feno apreendida, (bovinos 1-3 e 11), deixando, por vezes, o alimento pender da boca por longos períodos (Fig. 2). Essa forma de dificuldade de apreensão e mastigação resultava em salivação abundante (bovinos 3 e 11). Um animal (bovino 11) permanecia com a boca aberta por longo tempo. $\mathrm{O}$ bovino 4 mantinha a cabeça apoiada em objetos por longos períodos e o bovino 3 apresentou tremores musculares. O bovino 11, após um curso clínico de 9 dias de duração caiu ao ser movimentado, morrrendo poucos minutos após.

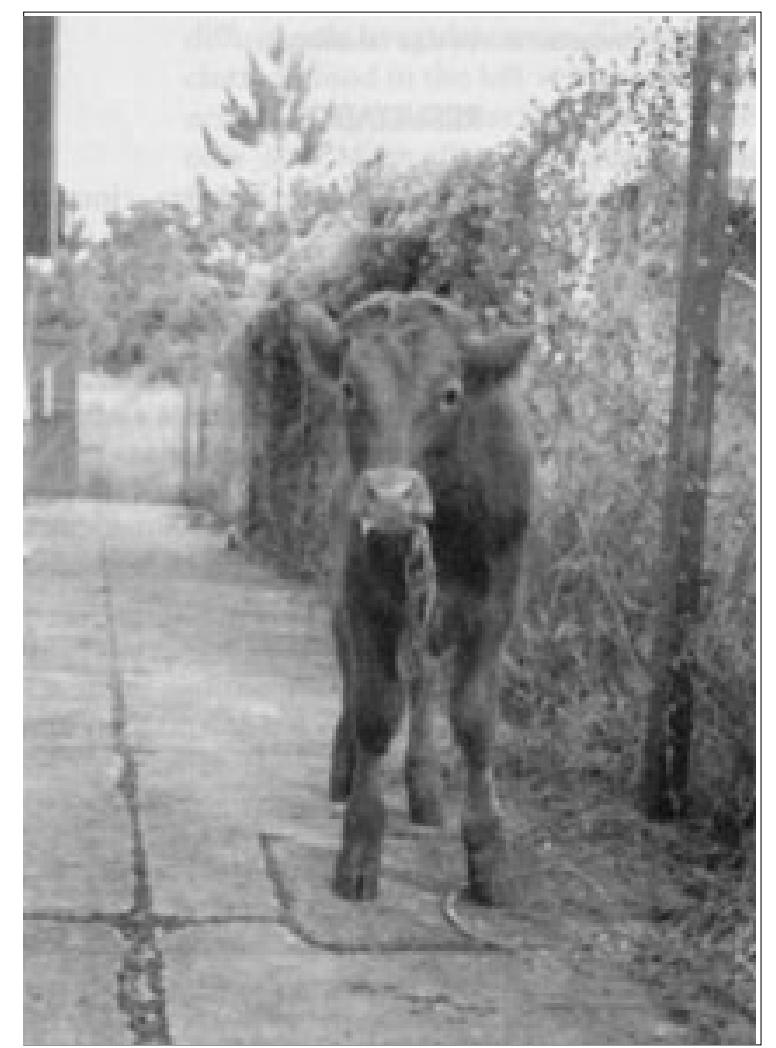

Fig. 1. Andar incoordenado e cruzamento dos membros posteriores ao andar, na intoxicação experimental por narasina. (bovino 3).

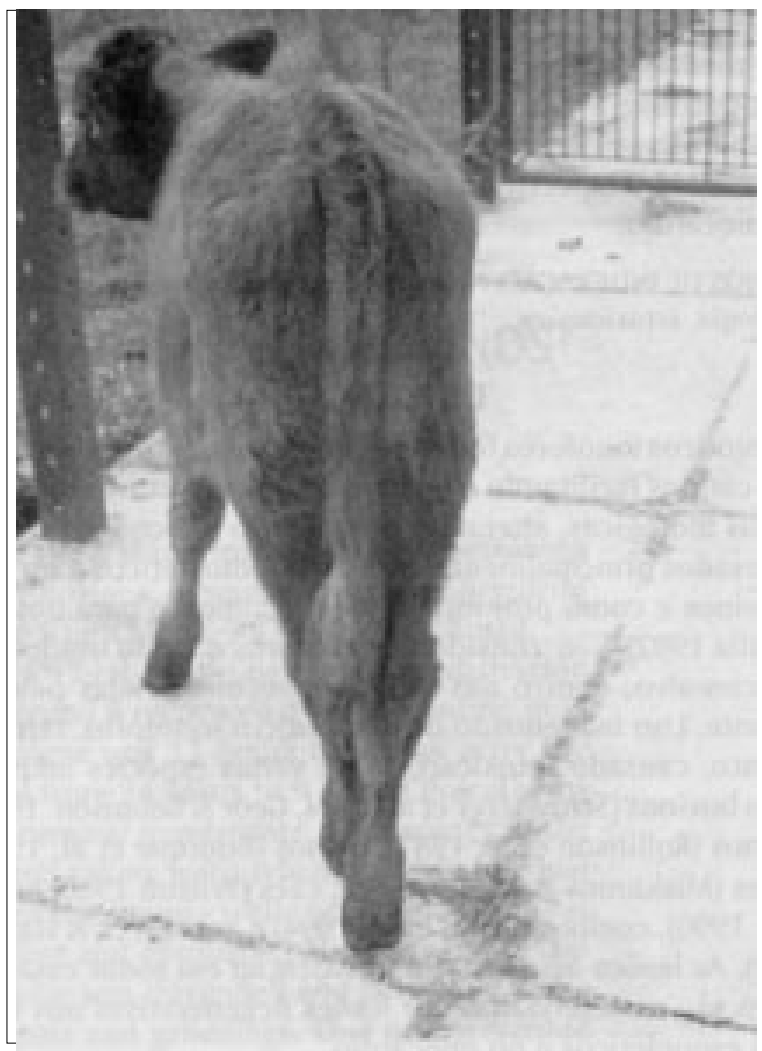

Fig. 2. Mastigação lenta. Porções do feno apreendido ficando longo tempo para fora da boca, na intoxicação experimental por narasina (bovino 3 ).

Sinais clínicos relacionados a distúrbios digestivos incluíram anorexia parcial ou total (bovinos 1, 2, 4, 11 e 12), diminuição da frequência e intensidade dos movimentos ruminais (bovinos 2 e 11) ou mesmo atonia ruminal (bovinos 1 e 12), fezes pastosas ou diarréicas (bovinos1, 4 e 11) e polidipsia (bovinos 3 e 12).

Alguns bovinos (3, 4 e 12) apresentaram sinais de distúrbios respiratórios. A respiração era abdominal nos bovinos 3 e 12, ofegante nos bovinos 3 e 4, e entrecortada no bovino 4. Outros sinais clínicos incluíam depressão (bovinos 1, 3 e 4), gemidos (bovinos 2, 3 e 4), aumento da intensidade dos sons cardíacos ( 1 e 4 ) e pulso positivo da veia jugular (bovino 1). $O$ bovino 3 apresentou mioglobinúria.

Os animais que se recuperaram tiveram quadro clínico leve, mais relacionado a distúrbios digestivos. Houve redução do apetite em um animal (bovino 6), diminuição da intensidade e freqüência dos movimentos ruminais em quatro (bovinos 6, 7, 8 e 9). As fezes eram pastosas em dois animais (bovinos 8 e 9), diarréicas em outros dois (bovinos 5 e 7) e ressequidas com muco em um outro (bovino 6). 0 bovino 7 apresentou diarréia intermitente por alguns dias, e após, fezes ressequidas e tenesmo. Nos bovinos que se recuperaram, os sinais clínicos de incapacitação muscular foram infrequentes e discretos. $O$ bovino 7 apresentou discreto andar incoordenado, e os bovinos 5 e 6 discreto arrastamento 

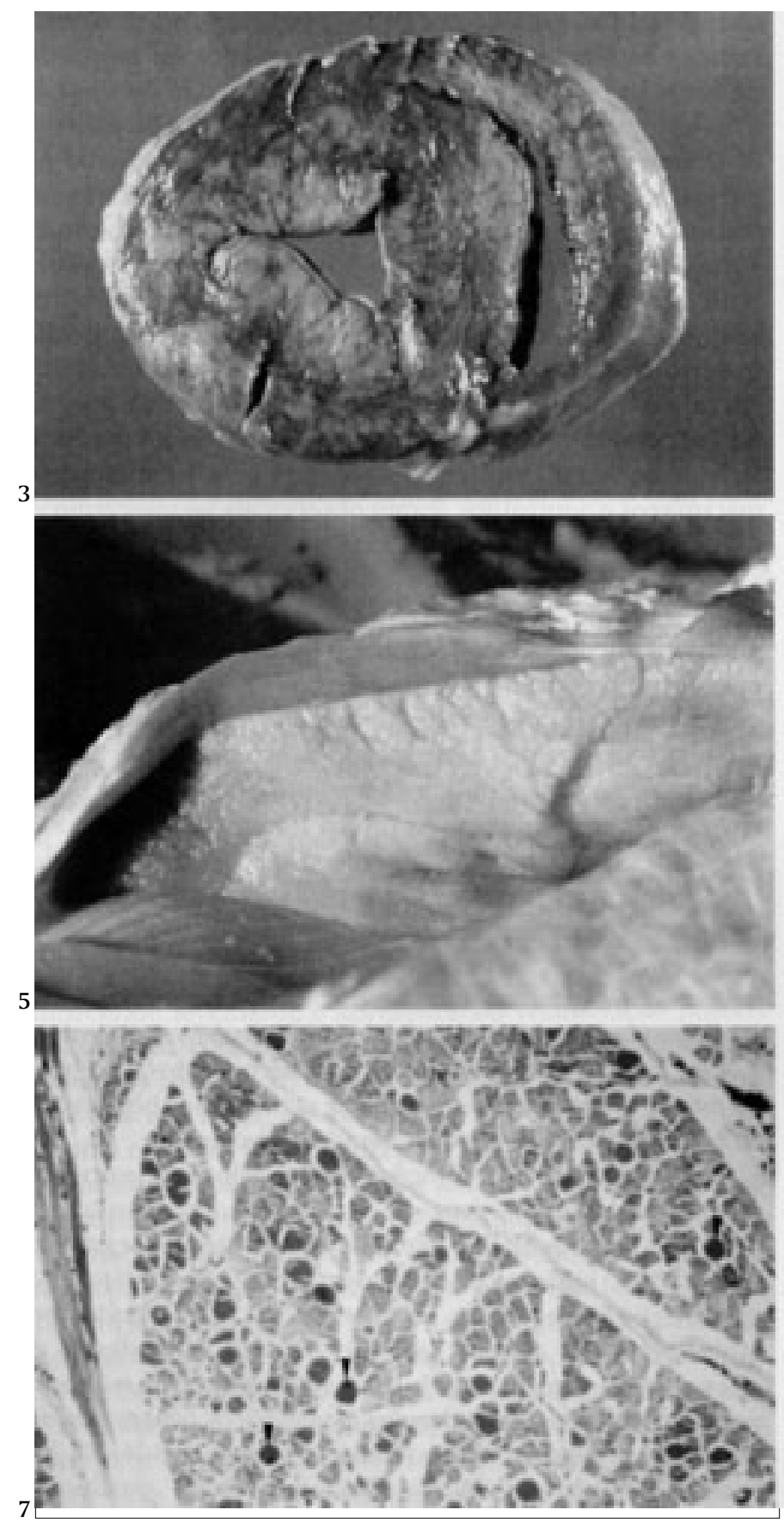

Fig. 3. Cardiomiopatia degenerativa acentuada (corte transversal do miocárdio mostra extensas áreas multifocais branco-amareladas), na intoxicação experimental por narasina (bovino 11).

Fig. 5. Miopatia degenerativa acentuada. Há extensas áreas amareladas no músculo semitendíneo, na intoxicação experimental por narasina (bovino 3).

Fig. 7. Necrose hialina segmentar de miofibras da língua, que perderam seus contornos multifacetados normais e estão arredondadas, eosinofílicas e com núcleos picnóticos (cabeças de setas), na intoxicação experimental por narasina (bovino 3). HE, obj. 2,5.
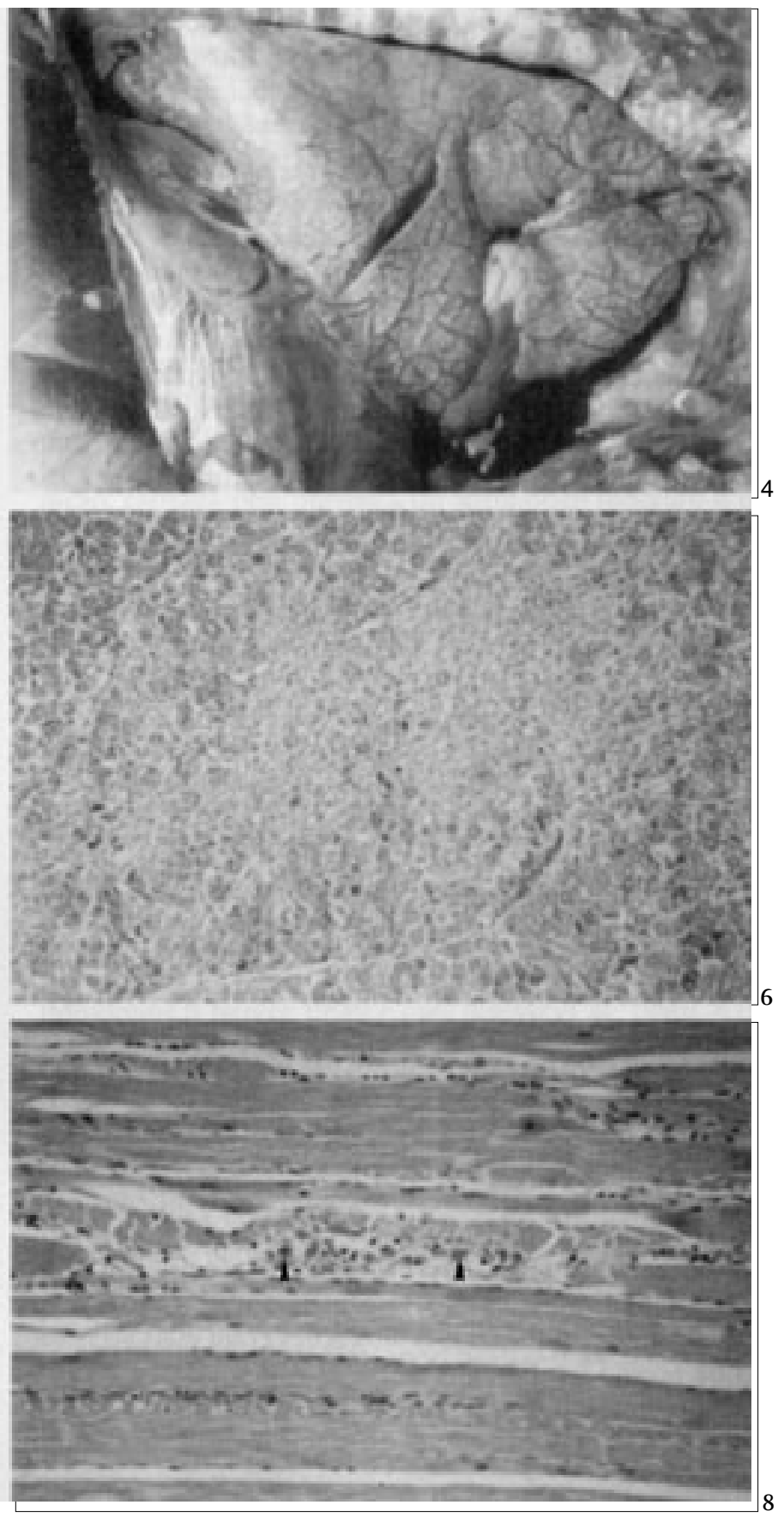

Fig. 4. Edema pulmonar intersticial. Os septos interlobulares das porções craniais do pulmão estão distendidos e têm aspecto gelatinoso, na intoxicação experimental por narasina (bovino 12).

Fig. 6. Área focal com extensa perda de miofibras e colapso do estroma do miocárdio, na intoxicação experimental por narasina (bovino 3). Tricrômico de Masson, obj. 2,5.

FIG. 8. Músculo vasto medial, necrose flocular segmentar. A miofibra ao centro da figura. está fragmentada e invadida por células satélites (cabeças de setas), na intoxicação experimental por narasina (bovino 12). HE, obj. 2,5. 
das pinças dos membros posteriores ao andar. Houve ainda aumento transitório da intensidade dos sons cardíacos no bovino 7 .

\section{Achados de necropsia}

As principais alterações encontradas na necropsia localizavam-se no miocárdio. Essas alterações foram observadas em cinco bovinos $(1,3,4,11$ e 12) e consistiam de áreas focais ou estrias múltiplas, pálidas, branco-amareladas, de contornos irregulares, distribuídas pelo miocárdio. Eram mais concentradas nas porções mais internas das paredes ventriculares, principalmente nos músculos papilares. Essas alterações foram acentuadas no bovino 11 (Fig. 3) moderadas no bovino 1 e 12 e discretas no bovino 4 . O ventrículo direito do bovino 3 apresentava palidez difusa discreta. No bovino 2 havia um grande coágulo de sangue no ventrículo esquerdo. Hemorragias petequiais multifocais subepicárdicas foram observadas no bovino 12.

Alterações decorrentes de insuficiência cardíaca também foram observadas em vários bovinos. Ocorreu hidrotórax nos bovinos 4 e 12 . Essa alteração era acentuada $(1.500 \mathrm{ml})$ no primeiro e moderada $(400 \mathrm{ml})$ no segundo. O líquido era citrino, coagulando minutos após a abertura da cavidade torácica. Edema pulmonar foi observado na necropsia de cinco bovinos. Era de distribuição intersticial nos bovinos 1, 4 e 12 e alveolar nos bovinos 2 e 11. O tipo intersticial (Fig. 4) caracterizava-se por dilatação dos septos e espaço subpleural por material semi-gelatinoso amarelado ou rosado. Essa alteração era acentuada nos bovinos 4 e 12 e moderada no bovino 1. Edema pulmonar do tipo alveolar era acentuado no bovino 11 e moderado no bovino 2 . Havia congestão pulmonar moderada no bovino 2 , edema subcutâneo da região submandibular no bovino 11, e rins congestos no bovino 4 .

Áreas pálidas foram observadas nos músculos esqueléticos de três bovinos (3, 4 e 12). Ocorriam com maior freqüência nos músculos masseter, extrínsecos da língua, tensor da fáscia lata, semitendíneo (Fig. 5), semimembranáceo, psoas, vasto lateral, reto da coxa, vasto medial, vasto intermédio, gastrocnêmio, músculos da face medial da escápula e no músculo grande dorsal. Essas alterações eram mais acentuadas no bovino 3 e bastante discretas nos bovinos 4 e 12 . A urina do bovino 3 estava acastanhada. Na cavidade oral dos bovinos 3 e 11 havia alimento ligeiramente mastigado compactado.

Lesões incidentais ou de menor importância foram também registradas. Os linfonodos do bovino 1 estavam aumentados de volume e suculentos ao corte. Havia hemorragias nos linfonodos pré-escapulares do bovino 2 . O conteúdo ruminal era líquido e abundante nos bovinos 11 e 12 . No bovino 3 observaram-se abscessos de aproximadamente 0,5 $\mathrm{cm}$ de diâmetro na parede do rúmen e edema moderado da submucosa das pregas do abomaso. $O$ conteúdo do intestino delgado era vermelho-acastanhado no bovino 4 e nódulos parasitários (cistos hidáticos) calcificados ou não foram vistos no fígado dos bovinos 3 e 11 .

\section{Achados histopatológicos}

Graus variáveis de tumefação e eosinofilia de miofibras, com perda das estriações e picnose nuclear, caracterizando necrose hialina multifocal, foram observados no miocárdio de todos os bovinos necropsiados, com exceção do controle e do bovino 2 . Em quatro bovinos $(1,3,11$ e 12) foi observada degeneração vacuolar caracterizada por formação de pequenos e grandes vacúolos no sarcoplasma de miofibras cardíacas. Necrose flocular, caracterizada por fragmentação do sarcoplasma necrótico com formação de agregados eosinofílicos irregulares ocorreu apenas no miocárdio do bovino 11 . No miocárdio desse animal havia infiltrado inflamatório composto de macrófagos, frequentemente com restos de material necrótico fagocitado no citoplasma, associado às lesões degenerativo-necróticas. Focos de infiltrado inflamatório misto, contendo linfócitos e, ocasionalmente, macrófagos e neutrófilos, foram observados no miocárdio de todos os bovinos. Edema rico em proteínas estava associado às lesões degenerativas no miocárdio dos bovinos 1 , 4 e 12 . Em dois bovinos (11 e 12) havia diversas áreas com perda de miofibras e colapso do estroma (Fig. 6). Essas áreas eram multifocais no bovino 11 e focal no bovino 12 . No miocárdio do bovino 11 foi observada fibrose discreta a moderada, e discreta tentativa de regeneração, caracterizada por células mesenquimais com citoplasma tumefeito e núcleos vesiculares envolvendo as miofibras. As lesões foram mais acentuadas nas seções de ventrículo esquerdo e septo interventricular. Nas seções correspondentes às aurículas e ao nodo sinoatrial não foram observadas lesões. O miocárdio de todos os bovinos necropsiados, inclusive o controle, apresentavam cistos de Sarcocystis sp.

Edema pulmonar intersticial foi observado nos bovinos 1,4 e 12 , com intensidade moderada no primeiro e acentuada nos dois outros. Edema alveolar ocorreu nos bovinos 2 e 11 e congestão pulmonar moderada no bovino 2 .

Graus variáveis de alterações histopatológicas ocorreram nos músculos esqueléticos. As alterações consistiam basicamente de necrose hialina (Fig. 7) e flocular (Fig. 8) segmentares, infiltrado inflamatório composto predominantemente por macrófagos e fenômenos regenerativos, caracterizados por mobilização de células satélites (Fig. 8) e formação de miotubos. Em menor grau havia também degeneração vacuolar. As lesões dos músculos esqueléticos ocorreram, em ordem decrescente de intensidade, nos bovinos 3, 4, 12, 1, 2 e 11. Os músculos mais afetados eram os do quadríceps femoral, semitendíneo e da língua; e os menos afetados o ocular e o longissimus dorsi. Lesões histológicas estavam presentes nos músculos com lesão macroscópica porém não incluídos na relação dos músculos sistematicamente colhidos para exame histológico. Estas eram acentuadas no bovinos 3 e discretas nos demais.

Havia congestão centrolobular discreta a moderada no fígado dos bovinos 1 e 11. Os hepatócitos tinham citoplasma vacuolizado nos bovinos 3, 4 e 11 e 12 . Essa alteração tinha distribuição centrolobular (bovinos 3, 4 e 12) ou difusa (bovino 11). Degeneração hidrópico-vesicular e microabscessos na mucosa do rúmen e edema moderado da submucosa do abomaso foram observados no bovino 3 . 


\section{DISCUSSÃO}

Narasina, em administrações orais, mostrou-se tóxica para bovinos. Doses de narasina iguais ou superiores a $15 \mathrm{mg} / \mathrm{kg}$ causaram a morte de bovinos. No entanto, doses semelhantes, de 14 ou $16 \mathrm{mg} / \mathrm{kg}$, quando fracionadas, respectivamente, em sete e quatro administrações diárias de 2 e $4 \mathrm{~m} / \mathrm{kg}$, provocaram somente sinais clínicos discretos seguidos de recuperação. Embora existam indicações de que os antibióticos ionóforos tenham poder acumulativo (Hulland 1993), isso não pôde ser confirmado nesse experimento.

O quadro clínico apresentado pelos bovinos intoxicados por narasina consistiu basicamente de alterações relacionadas a graus variáveis de incapacitação dos músculos esqueléticos e à insuficiência cardíaca. Um animal com curso clínico de 9 dias apresentou morte repentina após ter sido exercitado. Acontecimentos semelhantes têm sido relatados em surtos espontâneos da intoxicação por antibióticos ionóforos em bovinos (Shlosberg et al. 1992). Em um experimento de intoxicação por antibiótico ionóforo em ovinos (Wouters et al. 1997), o quadro clínico observado nessa espécie diferiu do observado nos bovinos deste estudo. Febre foi um sinal clínico frequiente em ovinos e não foi observado em bovinos. Sinais clínicos relacionados a distúrbios respiratórios ocorreram em aproximadamente $70 \%$ dos ovinos que receberam narasina e em menos de $30 \%$ dos bovinos intoxicados. Os sinais clínicos de distúrbios digestivos foram freqüentes nessas duas espécies. Ocorreram em todos os bovinos intoxicados e em cerca de $84 \%$ dos ovinos. Dos bovinos que adoeceram levemente e apresentaram recuperação posterior, os sinais clínicos mais freqüentes foram relacionados a distúrbios digestivos, representados por alterações na consistência das fezes, na intensidade e frequiência dos movimentos ruminais e no apetite.

Os distúrbios relacionados à incapacitação muscular observados nos bovinos intoxicados abrangeram alterações na locomoção, tais como andar incoordenado, relutância em mover-se, e também relacionados a apreensão, mastigação e deglutição de alimentos. Sinais semelhantes relacionados à locomoção têm sido descritos na intoxicação por outros antibióticos ionóforos em bovinos (Potter et al. 1984, Schweitzer et al. 1984, Geor \& Robinson 1985, Fourie et al. 1991), ovinos (Nation et al. 1982, Newsholme et al. 1983, Bourque et al. 1986, Gava 1991), suínos (Van Halderen et al. 1993), coelhos (Salles et al. 1994), cães (Karsai et al. 1990) e aves (Behr et al. 1988). Não se encontraram, no entanto, descrições de distúrbios relacionados à apreensão, à mastigação e à deglutição na intoxicação por antibióticos ionóforos em bovinos.

Os distúrbios respiratórios mais marcantes observados clinicamente nos bovinos deste experimento parecem não ter relação com a intensidade do edema pulmonar e foram, provavelmente, devidos à lesão nos músculos envolvidos na respiração, como diafragma e intercostais. Lesões do diafragma têm sido mencionadas para explicar distúrbios respiratórios em cavalos intoxicados (Doonan et al. 1987).

Diarréia ocorreu em cinco dos bovinos intoxicados. Este sinal clínico tem sido associado à intoxicação por antibióti- cos ionóforos em bovinos (Van Vleet et al. 1983, Geor \& Robinson 1985), mas a patogenia é incerta. É sugerido resulte de alteração iônica provocada pela droga ou por sua ação antimicrobiana (Bergen \& Bates 1984).

Anorexia, parcial ou total, foi um sinal clínico freqüente nos bovinos intoxicados e é tida como um dos sinais clínicos mais constantes na intoxicação por antibióticos ionóforos (Novilla 1992), tendo sido observada em associação a essa toxicose em bovinos (Van Vleet et al. 1983, Potter et al. 1984, Shlosberg et al. 1986), ovinos (Bourque et al. 1986, Fourie et al. 1991), suínos (Van Halderen et al. 1993), caninos (Karsai et al. 1990), equiinos (Rollinson et al. 1987) e coelhos (Salles et al. 1994). Alguns trabalhos sugerem que a perda parcial ou total do apetite seja devida a alterações na palatabilidade da ração, causada pela presença do antibiótico ionóforo e que funcionaria como um fator de defesa do animal, evitando a ingestão de quantidades maiores da droga (Shlosberg et al. 1986). No entanto, neste experimento e em outro realizado com narasina em ovinos (Wouters et al. 1997), em que a administração do antibiótico ionóforo não foi associada à ração, esses sinais clínicos provavelmente foram causados por alterações fisiopatológicas induzidas pela droga ou, alternativamente, pelo menos em alguns animais, à incapacidade em apreender alimentos, devido à lesão muscular.

Um bovino apresentou mioglobinúria macroscopicamente perceptível cinco dias após a administração da narasina. Mioglobinúria tem sido descrita em associação à intoxicação por antibióticos ionóforos em bovinos (Van Vleet et al. 1983), ovinos (Newsholme et al. 1983, Wouters et al. 1997), suínos (Miskimins \& Neiger 1996) e cães (Karsai et al. 1990). A presença de mioglobina na urina é indicativa de dano muscular grave (Hulland 1993).

Os resultados deste experimento, quando comparados com os de experimento semelhante com narasina realizado em ovinos (Wouters et al. 1997) sugerem que os ovinos são mais sensíveis que os bovinos. Doses de $4 \mathrm{mg} / \mathrm{kg}$ foram letais para ovinos e não causaram a morte de bovinos, mesmo quando administradas por 4 dias consecutivos.

Alterações macroscópicas foram encontradas principalmente no miocárdio. Eram áreas pálidas branco-amareladas de degeneração e estavam presentes em cinco dos seis bovinos intoxicados que foram necropsiados. Lesões semelhantes ocorreram nos músculos esqueléticos e foram observados em apenas três dos bovinos necropsiados, sendo pronunciadas em apenas um deles. Essa distribuição das lesões de necropsia é semelhante ao que é descrito na intoxicação em bovinos por outros antibióticos ionóforos (Van Vleet et al. 1983, Schweitzer et al. 1984, Galitzer et al. 1986, Shlosberg et al. 1986, Shlosberg et al. 1992).

Associadas às lesões do miocárdio ocorreram lesões de insuficiência cardíaca em outros órgãos. Edema pulmonar do tipo intersticial foi observado na necropsia de 3 bovinos. Outras alterações relacionadas a insuficiência cardíaca foram observadas na necropsia de 4 bovinos e caracterizavam-se por hidrotórax, edema submandibular, congestão renal e congestão e edema alveolar pulmonares. Um outro bovino apresentou um grande coágulo sanguíneo no ventrículo es- 
querdo, e tinha também congestão e edema pulmonares acentuados, não apresentando, no entanto, lesões macroscópicas e histológicas no miocárdio. A presença de coágulo sangüíneo no ventrículo esquerdo é indicativa de degeneração miocárdica acentuada (Robinson \& Maxie 1993). Nesse caso, como o bovino apresentou curso clínico agudo (morreu cerca de 60 horas após a administração de narasina) o sinal de insuficiência cardíaca deve ser atribuído a distúrbios funcionais ou bioquímicos no miocárdio. Em um bovino deste experimento, a morte foi precipitada após exercício. A morte desse animal foi atribuída à insuficiência cardíaca. Esse bovino apresentou marcada cardiomiopatia degenerativa. Observações semelhantes são também relatadas em estudos realizados com outros antibióticos ionóforos (Galitzer et al. 1986).

Os achados histopatológicos nos bovinos deste experimento consistiram basicamente de miopatia e cardiomiopatia degenerativas. Embora lesões macroscópicas indicativas de degeneração de músculos esqueléticos estivessem presentes somente em três bovinos, e eram discretas em dois deles, todos os bovinos intoxicados que foram necropsiados apresentavam alterações histológicas de miopatia degenerativa e cinco deles apresentavam alterações microscópicas no miocárdio. Quando comparados, no entanto, os graus de intensidade das lesões histológicas encontradas no músculos esqueléticos, percebe-se que a intensidade das lesões dos músculos esqueléticos foi superior à média das alterações histológicas encontradas no miocárdio.

Processos reparativos no miocárdio estavam presentes em apenas um bovino, justamente o animal que apresentou $o$ curso clínico mais longo. A intensidade dos fenômenos reparativos nos músculos esqueléticos não foram, no entanto, sempre proporcionais à duração do curso clínico. Um bovino que apresentou lesões reparativas acentuadas no miocárdio, tinha uma das menores intensidades de reparação nos músculos esqueléticos, quando comparado aos demais bovinos necropsiados.

\section{REFERÊNCIAS}

Behr K-P., Lüders H., Glünder G. \& Friedrichs M. 1988. Narazin-Intoxikation bei Puten. Dtsch. Tierärztl. Wschr. 95(3):107-111.

Bergen W.G. \& Bates D.B. 1984. Ionophores: their effect on production efficiency and mode of action. J. Anim. Sci. 58(6):1465-1483.

Bourque J.G., Smart M. \& Wobeser G. 1986. Monensin toxicity in lambs. Can. Vet. J. 27: 397-399.

Doonan G.R., Brown C.M., Mullaney T.P., Brooks D.B., Ulmanis E.G. \& Slanker M.R. 1989. Monensin poisoning in horses - an international incident. Can. Vet. J. 30(2):165-169.

Fourie N., Bastianello S.S., Prozesky L., Nel P.W. \& Kellerman T.S. 1991.
Cardiomyopathy of ruminants induced by litter of poultry fed on rations containing the ionophore antibiotic maduromicin. I. Epidemiology, clinical signs and clinical pathology. Onderstepoort J. Vet. Res. 58: 291296.

Galitzer S.J., Kruckenberg S.M. \& Kidd J.R. 1986. Pathologic changes associated with experimental lasalocid and monensin toxicosis in cattle. Am. J. Vet. Res. 47(12):2624-2626.

Gava A. 1991. Comunicação pessoal (UDESC-CAV, Lages, Santa Catarina).

Geor R. J. \& Robinson W.F. 1985. Suspected monensin toxicosis in feedlot cattle. Aust. Vet. J. 62: 130-131.

Hulland T.J. 1993. Muscle and tendon, p. 183-265. In: Jubb K.V.F., Kennedy P.C., Palmer N. (ed.). Pathogy of Domestic Animals. Vol. 1. 4th ed. Academic Press, San Diego. 780 p.

Karsai F., Papp L., Sályi G., Bagó Gy. \& Kántás K. 1990. Gehäuft auftretende Narazin-Vergiftung bei Hunden. Tierärztl. Umschau 45: 316-324.

Miskimins D.W. \& Neiger R.D. 1996. Monensin toxicosis in swine. J. Vet. Diagn. Invest. 8: 396-397.

Nation P.N., Crowe S.P. \& Harries W.N. 1982. Clinical signs and pathology of accidental monensin poisoning in sheep. Can. Vet. J. 23: 323-326.

Newsholme S.J., Howerth E.W., Bastianello S.S., Prozesky L. \& Minné J.A. 1983. Fatal cardiomyopathy in feedlot sheep attributed to monensin toxicosis. J. South. Afr. Vet. Assoc. 54: 29-32.

Novilla M.N. 1992. The veterinary importance of the toxic syndrome induced by ionophores. Vet. Hum. Toxicol. 34: 66-70.

Potter E.L., Van Duyn R.L. \& Colley C.O. 1984. Monenisn toxicity in cattle. J. Anim. Sci. 58(6):1499-1511.

Robinson W.F. \& Maxie M.G. 1993. The cardiovascular system, p. 1-100. In: Jubb K.V.F., Kennedy P.C. \& Palmer N. (ed.) Pathology of Domestic Animals. Vol. 3. 4th ed. Academic Press, San Diego.

Rollinson J., Taylor F.G.R. \& Chesney J. 1987. Salinomycin poisoning in horses - an international incident. Vet. Rec. 121: 126-128.

Salles M.S, Lombardo de Barros C.S. \& Barros S.S. 1994. Ionophore antibiotic (narasin) poisoning in rabbits. Vet. Hum. Toxicol. 36(5):437-444.

Schweitzer D., Kimberling C., Spraker T., Sterner F.E., McChesney A.E.. 1984. Accidental monensin sodium intoxication of feedlot cattle. J. Am. Vet. Med. Assoc. 184(10):1273-1276.

Shlosberg A., Harmelin A., Perl S., Pano G., Davidson M., Orgad U., Kali U., Bor A., Van Ham M., Hoida G., Yacobson B., Avidar Y., Israeli B-A. \& Bogin E. 1992. Cardiomyopathy in cattle induced by residues of the coccidiostatic maduramicin in poultry litter given as a feedstuff. Vet. Res. Comm. 16(1):45-58.

Shlosberg A., Perl S., Yakobson B., Klopfer U., Egyed M.N., Nobel T.A. \& Efron Y. 1986. The chronic course of a probable monensin toxicosis in cattle. Vet. Hum. Toxicol. 28: 230-233.

Van Halderen A., Bastianello S.S., Fourie N. \& Zumpt I.F. 1993. An outbreak of narasin poisoning in swine. J. South Afr. Vet. Assoc. 64(1):43-46.

Van Vleet J.F., Amstutz H.E., Weirich W.E., Rebar A.H. \& Ferrans V.J. 1983. Clinical, clinicopathologic, and pathologic alterations in acute monensin toxicosis in cattle. Am. J. Vet. Res. 44: 2133-2144.

Wouters F., Wouters A.T.B. \& Barros C.S.L. 1997. Intoxicação experimental por narasina em ovinos. Pesq. Vet. Bras. 17. (No prelo) 